\title{
Expression and serological diagnosis of Mycobacterium tuberculosis CFP-10 and Rv2626c proteins
}

\author{
Z.Y. Zhu' ${ }^{1}$, D. Zhang' ${ }^{2}$ H.B. Wang ${ }^{1}$, J.Z. Xiao' ${ }^{1}$, Y.F. Qiu ${ }^{3}$, L. Yan ${ }^{3}$, \\ D. Chen ${ }^{3}$, A.G. Liu $^{3}$ and X. Yang ${ }^{3}$ \\ ${ }^{1}$ Hainan Provincial Nongken General Hospital, Haikou, Hainan, China \\ ${ }^{2}$ College of Environment and Plant Protection of Hainan University, Haikou, \\ Hainan, China \\ ${ }^{3}$ Nanjing Potomac BioTechnology Co. Ltd., Nanjing, Jiangsu, China \\ Corresponding author: Z.Y. Zhu \\ E-mail: zhuzhongyuan197@163.com
}

Genet. Mol. Res. 13 (3): 7398-7406 (2014)

Received June 18, 2013

Accepted March 14, 2014

Published September 12, 2014

DOI http://dx.doi.org/10.4238/2014.September.12.5

\begin{abstract}
We constructed a Mycobacterium tuberculosis vector expressing CFP-10 and Rv2626c to examine the expression of these proteins in Escherichia coli as well as their immunoreactivity. The CFP-10 and Rv2626c genes were amplified from tuberculosis H37Rv genomic DNA using polymerase chain reaction. They were ligated into the expression vector PET30a and expressed in E. coli. Histidine tag nickel column chromatography was used to purify the recombinant protein. An enzyme-linked immunosorbent assay (ELISA) was used for detection. In our E. coli-engineered bacteria containing a CFP10 and Rv2626c plasmid, the target protein was found mainly to be in the soluble form. We formed mixed antigens of the recombinant CFP10 and Rv2626c proteins. ELISA results showed that in 214 blood samples, the positive rate was $77.1 \%$. The target gene was successfully expressed in the host strain. Mixed antigens of the recombinant CFP-
\end{abstract}


10 and Rv2626c proteins can be used as a combination antigen in the serological diagnosis of tuberculosis.

Key words: Mycobacterium tuberculosis; CFP-10 protein; ELISA; Rv2626c protein; Serodiagnosis

\section{INTRODUCTION}

Tuberculosis (TB) has the highest mortality rate caused by a single pathogen infection worldwide, making it a serious threat to human health. Approximately one-third of people have been infected with TB, and new infections are increasing at an annual rate of 7-8 million. Therefore, rapid and accurate diagnosis of TB is important for controlling the TB epidemic. Currently, TB diagnosis in China primarily depends on clinical symptoms, combined diagnostic imaging and sputum smear microscopy, bacterial culture, and a purified protein derivative skin test. However, the sensitivity of conventional bacteriological examination methods is low and a long period of time is required for bacterial culture (6-8 weeks). Human actions, among other reasons, limit the use of rapid TB tests (Weldingh et al., 2005). The enzymelinked immunosorbent assay (ELISA) to detect the TB antibody levels is simple, inexpensive, and fast. TB secretes proteins into the extracellular environment and plays an important role in the immune response to TB. Studies have shown that CFP10 is a target antigen of T cells and that it can induce a delayed hypersensitivity skin reaction as well as stimulate peripheral blood mononuclear cells to produce specific interferon- $\gamma$ (Dillon et al., 2000). Rv2626c antigens are specific proteins expressed in dormant Mycobacterium tuberculosis (MTB) and show a strong antigen response, which can stimulate the body to produce higher levels of antibodies (Rosenkrands et al., 2002). In this study, we constructed a TB CFP-10 and Rv2626c protein expression vector and expressed these proteins in Escherichia coli BL21 (DE3) cells. Expressed products were purified to obtain a large amount of CFP-10 antigen and Rv2626c antigen. Recombinant CFP-10 and Rv2626c protein antigens were combined and tested using an ELISA to determine whether the recombinant protein could be applied for the serological diagnosis of TB.

\section{MATERIAL AND METHODS}

\section{Strains and vectors}

M. tuberculosis H37Rv, E. coli BL21 (DE3), and the pET-30a expression vector were stored in Hainan Land Reclamation General Hospital.

\section{Reagents}

$\beta$-Thiogalactoside (IPTG) and kanamycin (Kan) were purchased from Shanghai Sangon Biological Engineering Technology \& Services Ltd. (Shanghai, China). DNA polymerase, T4 DNA ligase, restriction endonucleases, and the DNA marker were purchased from Takara (Dalian) Co., Ltd. (Shiga, Japan). The pre-stained protein marker was purchased from Beijing Bai Tai Ke Biotechnology Co., Ltd. (Beijing, China). The agarose gel DNA 
extraction kit was purchased from Beijing Tian Gen Biotechnology Co. (Beijing, China). The 6X His-tagged Ni-NTA agarose protein purification column was from QIAGEN (Hilden, Germany). Common high-purity biochemical reagents were from Biosharp (Hefei, China). Other reagents were of analytical grade. Horseradish-peroxidase (HRP)-labeled anti-human immunoglobulin (Ig) was purchased from Solarbio Science and Technology Co., Ltd. (Beijing, China). The chromogenic substrate was purchased from InTec Technology Limited (Xiamen, China).

\section{Source of serum}

A total of 118 blood samples from TB patients were provided by the Haikou Tuberculosis Control and Treatment Department. Twenty patients had positive sputum smear results. Ninety-eight patients had a negative sputum smear. The serum from 96 healthy subjects was from Hainan Land Reclamation General Hospital.

\section{Primers}

Based on the CFP-10 and Rv2626c gene sequences in standard strains of M. tuberculosis $\mathrm{H} 37 \mathrm{Rv}$ shown in GenBank, the 5' primer I sequence of the CFP-10 gene was 5'-CCGGA ATTCATGGCAGAGATGAAGACCGAT-3', while the 3' primer II sequence was 5'-CCCAA GCTTTCAGAAGCCCATTTGCGAGGAC-3'. The restriction sites for primers I and II were EcoRI and HindIII. The Rv2626c gene 5' primer III sequence was: 5'-CCGGAATTCATGACC ACCGCACGCGACA-3', while the 3' end primer IV sequence was: 5'-CCGCTCGAGCTAG CTGGCGAGGGCCAT-3'. The restriction sites for primers III and IV were EcoRI and XhoI. Primers were synthesized by GenScript, Ltd. (Piscataway Township, NJ, USA).

\section{CFP-10 and Rv2626c gene amplification and cloning}

H37Rv genomic DNA was used as a template and polymerase chain reaction (PCR) was used to amplify CFP-10 and Rv2626c gene fragments. The reaction conditions were as follows: $95^{\circ} \mathrm{C}$ for $5 \mathrm{~min}, 95^{\circ} \mathrm{C}$ for $30 \mathrm{~s}, 53^{\circ} \mathrm{C}$ for $30 \mathrm{~s}$, and $72^{\circ} \mathrm{C}$ for $1 \mathrm{~min}$ for $36 \mathrm{cycles}$; this was followed by $72^{\circ} \mathrm{C}$ for $7 \mathrm{~min}$, and maintained at $4^{\circ} \mathrm{C}$ for preservation. PCR products were processed using the purification kit. After restriction enzyme digestion, T4 DNA ligase was used to ligate the fragments into the $\mathrm{pET} 30 \mathrm{a}$ vector. Ligation products were transformed into E. coli BL21 (DE3) competent cells, and positive recombinants were selected for sequencing by Nanjing GenScript Biological Engineering Co., Ltd. (Nanjing, China).

\section{Induced expression of target protein}

The sequencing-verified recombinants were inoculated into Luria-Bertani (LB) liquid medium containing $50 \mathrm{mg} / \mathrm{L} \mathrm{Kan}$. After shaking the culture at $37^{\circ} \mathrm{C}$, the absorbance $\left(\mathrm{A}_{600}\right)$ was adjusted to 0.6-0.8. IPTG was added to reach a final concentration of $1 \mathrm{mM}$, and the culture was continued at $37^{\circ} \mathrm{C}$. After $1 \mathrm{~h}$ of expression induction, the samples were collected at 1 -h intervals until $6 \mathrm{~h}$. Bacterial cells were harvested for sodium dodecyl sulfate-polyacrylamide gel electrophoresis (SDS-PAGE) analysis. 


\section{Expression, identification, and purification of recombinant proteins}

The bacterial cells were collected according to the best conditions for inducible expression. After cooling in an ice bath, sonication, and centrifugation, the pellet and supernatant were collected. SDS-PAGE was used to analyze target protein expression. A QIAGEN 6X His-tagged Ni-NTA agarose protein purification column was used to purify His-tagged recombinant proteins.

\section{Recombinant CFP-10 and Rv2626c protein antigens by ELISA}

Using the matrix method, the optimal antigen coating concentration and optimal second antibody dilutions were determined. In $\mathrm{pH} 9.6$ coating buffer, the recombinant CFP-10 protein, Rv2626c protein, and mixture of both proteins were diluted to $2,1,0.5,0.1$, and 0.01 $\mu \mathrm{g} /$ well. A $100-\mu \mathrm{L}$ sample was added per well at $4^{\circ} \mathrm{C}$ coating and the plate was incubated overnight. The next day, the remaining liquid in each well was dried and the well was washed 3 times. Calf serum was diluted 10 -fold, and $200 \mu \mathrm{L}$ sample was added per well; the plate was sealed and incubated at $37^{\circ} \mathrm{C}$ for $1 \mathrm{~h}$. The wells were dried and washed 3 times with phosphate buffer solution. Serum samples were diluted 1:50 with phosphate-buffered saline. Then, 100 $\mu \mathrm{L}$ was added to the wells and the plate was placed at $37^{\circ} \mathrm{C}$ for $1.5 \mathrm{~h}$. The remaining liquid was dried in the wells and the wells were washed 3 times with washing liquid. Horseradish peroxidase (HRP)-secondary antibody was diluted 1:500, 1:1000, and 1:1500, and $100 \mu \mathrm{L}$ sample was added per well; the plate was placed at $37^{\circ} \mathrm{C}$ for $1 \mathrm{~h}$. After washing 3 times, $50 \mu \mathrm{L}$ substrate solutions A and B were added to each well for 15 min for the dark reaction. Next, 1

$\mathrm{M} \mathrm{HCl}$ was added to stop the reaction, and the optical density at $450 \mathrm{~nm}\left(\mathrm{OD}_{450}\right)$ was measured using a microplate reader. $\mathrm{OD}_{450} \geq 2.1$ times of the negative control value was defined as positive; sensitivity and specificity were also calculated.

\section{RESULTS}

\section{Recombinant CFP-10 and Rv2626c plasmid identification}

Recombinant clones were chosen from the constructed positive recombinant bacteria. After incubation, plasmids were extracted for double-digestion identification (Figure 1). The recombinant plasmid fragments and PCR fragments were identical and were approximately 303 and $432 \mathrm{bp}$ in length. Sequencing of the recombinant plasmid showed that the sequence was genetically identical to that of M. tuberculosis H37Rv in GenBank.

\section{Induced expression of recombinant plasmid in E. coli}

The screened pET-30a-CFP-10/BL21 (DE3) and pET-30a-Rv2626c/BL21 (DE3) were induced with IPTG. Bacterial cell sedimentation was used for SDS-PAGE analysis (Figure 2). Specific expression product bands were visible. The recombinant CFP-10 protein had a relatively larger molecular mass than expected, which may be because the additional sequence in the vectors was excessively expressed. The relative molecular mass of recombinant Rv2626c protein was consistent with the results expected, while non-induced bacterial cells showed no significant bands. 


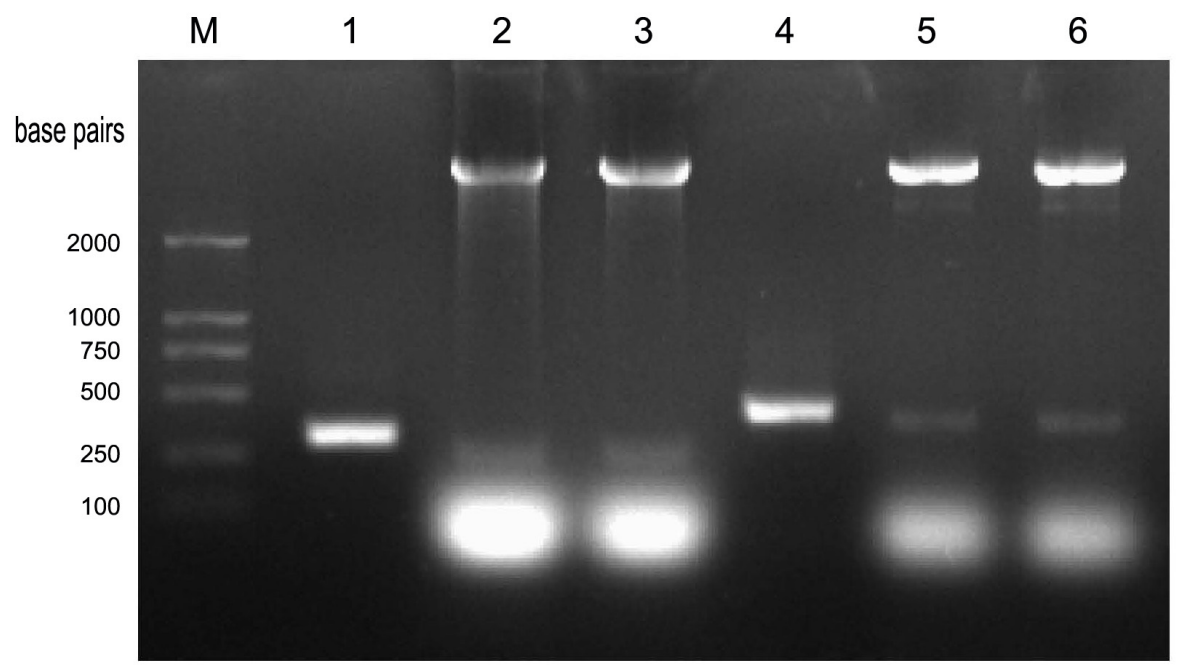

Figure 1. Results of restriction digestion of recombinant plasmids pET30a-CFP-10 and pET30a-Rv2626c. Lane $M$ $=$ DNA marker; lane $1=\mathrm{CFP}-10$ gene PCR product; lanes 2 and $3=$ pET-30a-CFP-10 double-digestion; lane $4=$ Rv2626c gene PCR product; lanes 5 and $6=$ pET-30a-Rv2626c pair fragment.

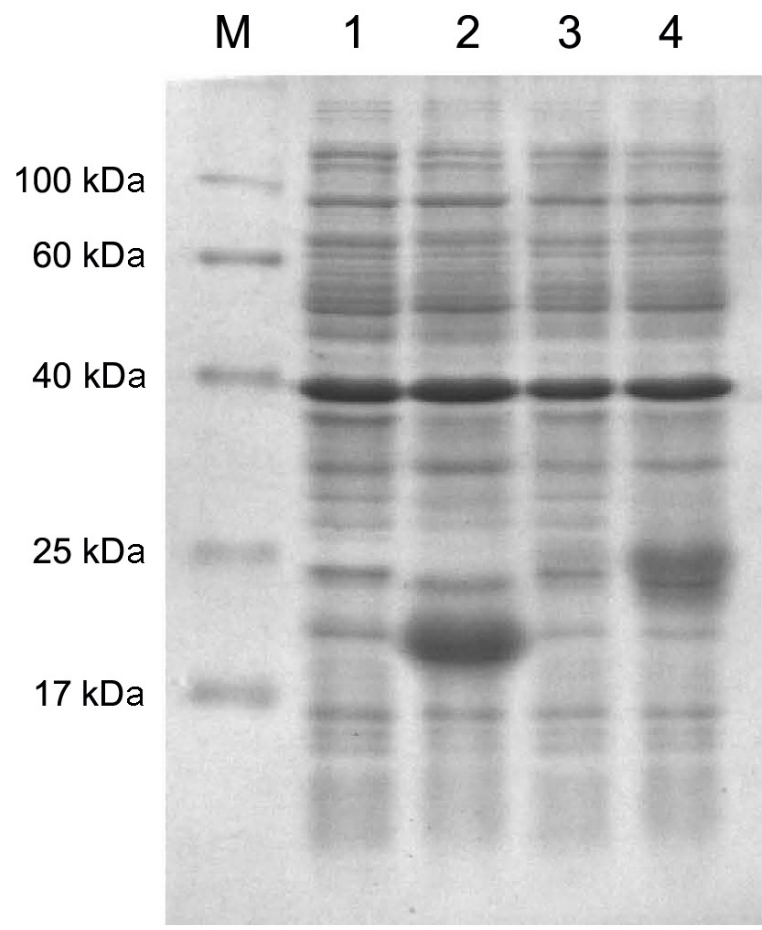

Figure 2. SDS-PAGE of recombinant expression products. Lane $M=$ pre-stained protein marker; lane 1 $=$ uninduced recombinant CFP-10 strain; lane $2=6 \mathrm{~h}$ after induction of recombinant CFP-10 strain; lane $3=$ uninduced recombinant Rv2626c strains; lane $4=6 \mathrm{~h}$ after induction of recombinant Rv2626c strain. 


\section{Soluble analysis of recombinant CFP-10 and RV2626c protein}

Induced bacterial cells were sonicated and centrifuged and the supernatant and pellet were examined by SDS-PAGE. The results are shown in Figure 3. Recombinant CFP-10 and Rv2626c proteins were primarily present in the supernatant and were soluble.

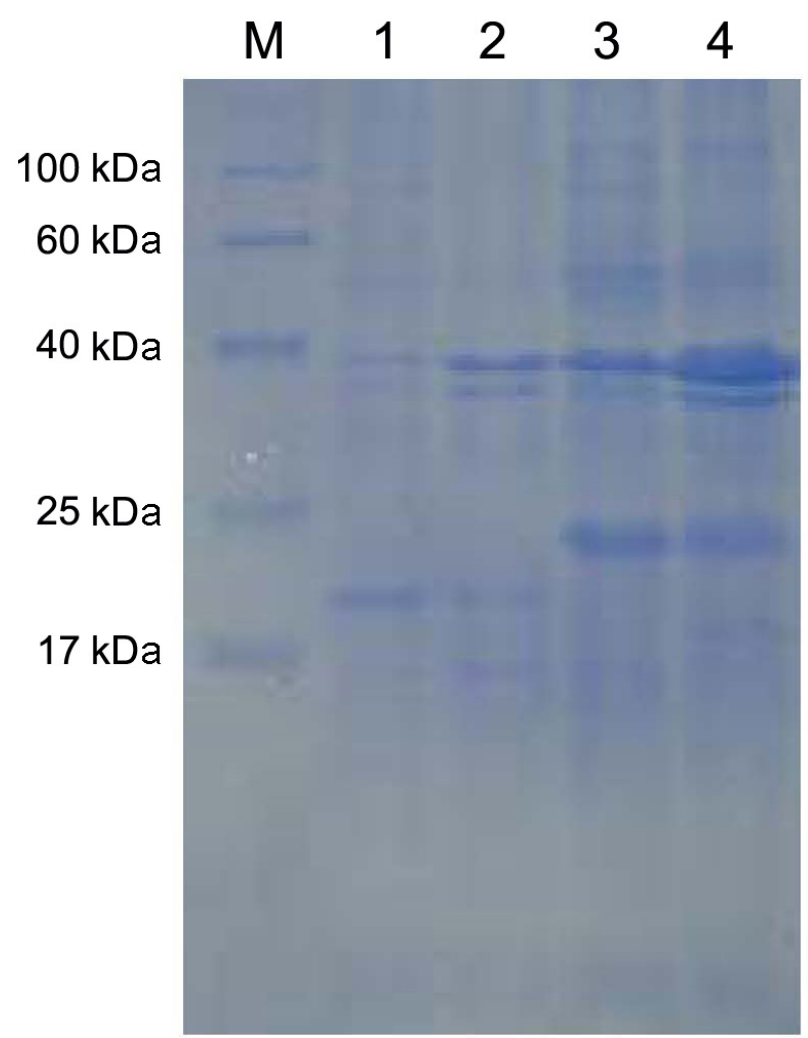

Figure 3. Expression analysis of recombinant CFP-10 and Rv2626c peptides. Lane $M=$ protein molecular weight standards; lane 1 = recombinant CFP-10 protein cell supernatant after sonication; lane $2=\mathrm{CFP}-10$ protein in recombinant bacteria precipitate after sonication; lane $3=$ recombinant protein Rv2626c cell supernatant after sonication; lane 4 = recombinant protein Rv2626c precipitate after cell sonication.

\section{Purification of recombinant CFP-10 protein}

Induced bacteria cells were collected and sedimented, after which the cells were sonicated and centrifuged. The supernatant was purified by Ni-agarose gel column purification. The elution peak solution was collected for SDS-PAGE analysis (Figure 4).

\section{ELISA}

Using the matrix method, the optimal coating concentration of CFP-10 peptides was 
found to be $0.1 \mu \mathrm{g} / \mathrm{well}$, Rv2626c protein antigens were $0.1 \mu \mathrm{g} / \mathrm{well}$, and the optimal concentration of mixed antigens was $0.1 \mu \mathrm{g} /$ well. The ratio of these values was 1:1. The optimal working concentration of HRP secondary antibody was 1:1500. The recombinant CFP-10 protein, Rv2626c protein, and mixture of the 2 antigens were used to examine serum from 118 patients and 96 healthy controls. The results showed that the overall sensitivity of the recombinant protein antigen CFP-10 for TB-specific antibodies was $75.4 \%$ (70.0\% in sputum smearpositive samples vs $76.5 \%$ in sputum smear-negative samples). Serum specificity in healthy subjects was $85.4 \%$ (82/96). The sensitivity of recombinant Rv2626c protein antigen-specific antibody for TB detection was $44.1 \%$ (45.0\% in sputum smear-positive samples $v s 43.9 \%$ in sputum smear-negative samples). Serum specificity in healthy subjects was $75.0 \%(72 / 96)$. Overall sensitivity of mixed antigen-specific antibodies for TB detection was $77.1 \%(80.0 \%$ in sputum smear-positive samples vs $76.5 \%$ in sputum smear-negative samples). Serum specificity in healthy subjects was $82.3 \%(79 / 96)$.

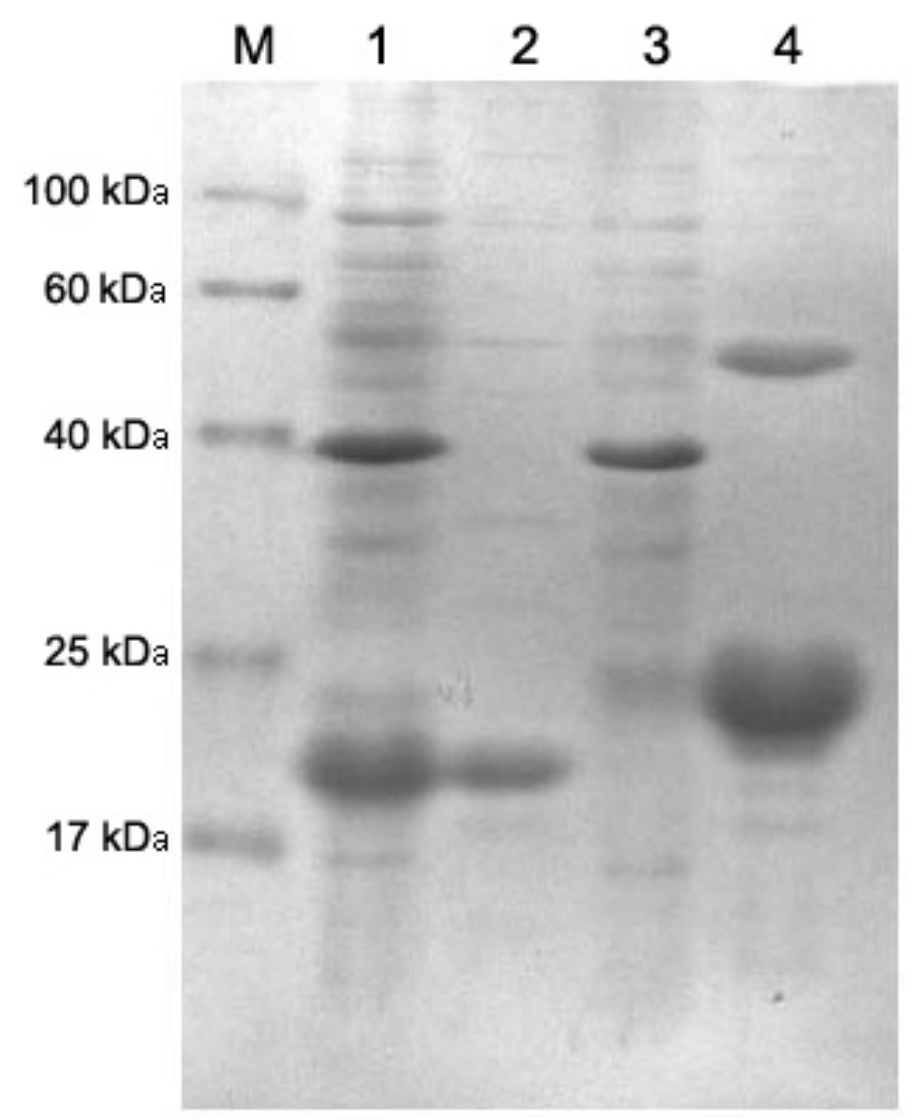

Figure 4. SDS-PAGE of purified recombinant CFP-10 protein and recombinant Rv2626c protein. Lane $M=$ protein molecular weight standards; lane $1=$ recombinant protein isolates induced CFP-10 product; lane 2 = purified recombinant CFP-10 protein; lane $3=$ strains induced by recombinant Rv2626c protein product; lane $4=$ purified recombinant Rv2626c protein. 


\section{DISCUSSION}

CFP10 is a low-molecular weight protein identified in 1998 and is also known as mtbll. It is a short-term culture filtrate protein encoded by the $\operatorname{lh} p$ gene and belongs to the ESAT- 6 family (Berthet et al., 1998). The size of the CFP10 gene is $303 \mathrm{bp}$ and the gene encodes 100 amino acids to produce a protein of $10 \mathrm{kDa}$. This protein exists only in the $M$. tuberculosis complex and several other pathogenic mycobacteria; Bacille Calmette-Guérin and other mycobacteria do not express CFP10 (Lewis et al., 2003). Therefore, as an antigen, CFP10 can distinguish TB, Bacille Calmette-Guérin immunization, and TB infection. Renshaw et al. (2002) found that CFP-10 shows an equivalent T-cell immune response to ESAT-6, which has potential diagnostic value.

The function of the Rv2626c antigen, which is specifically expressed during MTB dormancy, is unclear. The Rv2626c gene is 432 bp and encodes 143 amino acids; its expression is controlled by dormancy regulon (DosR). Expression of the gene is an important landmark for MTB to enter dormancy. A previous study found that the Rv2626c gene and downstream gene expression could cause MTB to enter dormancy and evade the host immune system (Park et al., 2003). Rv2626c antigens can stimulate the body to produce higher levels of antibodies. Therefore, the Rv2626c antigen can be used for serological diagnosis to identify MTB infection in the host organism.

In this study, we used genetic engineering to obtain the desired gene and constructed a prokaryotic vector expressing the CFP-10 and Rv2626c proteins. Recombinant proteins were successfully expressed in E. coli and were subsequently purified in high yield. This study provides a foundation for using prophase antigens to examine restructuring peptides for application in the serological diagnosis of TB

In this study, ELISA was used to detect recombinant CFP-10 and Rv2626c proteins and IgG antibodies of the 2 mixed antigens. The sensitivity of the combined antigen-specific antibodies for TB detection was $77.1 \%$, while the detection specificity in healthy control subjects was $82.3 \%$. Experimental data showed that a combination of the two methods were highly sensitive and specific. Further studies should be conducted to optimize the purification conditions and protein stability. These proteins show promise for use in clinical applications.

\section{ACKNOWLEDGMENTS}

Research supported by the Hainan Key Technology Projects in 2008 (\#080209).

\section{REFERENCES}

Berthet FX, Rasmussen PB, Rosenkrands I, Andersen P, et al. (1998). A Mycobacterium tuberculosis operon encoding ESAT-6 and a novel low-molecular-mass culture filtrate protein (CFP-10). Microbiology 144 (Pt 11): 3195-3203.

Dillon DC, Alderson MR, Day CH, Bement T, et al. (2000). Molecular and immunological characterization of Mycobacterium tuberculosis CFP-10, an immunodiagnostic antigen missing in Mycobacterium bovis BCG. J. Clin. Microbiol. 38: 3285-3290.

Lewis KN, Liao R, Guinn KM, Hickey MJ, et al. (2003). Deletion of RD1 from Mycobacterium tuberculosis mimics bacille Calmette-Guerin attenuation. J. Infect. Dis. 187: 117-123.

Park HD, Guinn KM, Harrell MI, Liao R, et al. (2003). Rv3133c/dosR is a transcription factor that mediates the hypoxic response of Mycobacterium tuberculosis. Mol. Microbiol. 48: 833-843.

Renshaw PS, Panagiotidou P, Whelan A, Gordon SV, et al. (2002). Conclusive evidence that the major T-cell antigens 
of the Mycobacterium tuberculosis complex ESAT-6 and CFP-10 form a tight, 1:1 complex and characterization of the structural properties of ESAT-6, CFP-10, and the ESAT- $6 *$ CFP-10 complex. Implications for pathogenesis and virulence. J. Biol. Chem. 277: 21598-21603.

Rosenkrands I, Slayden RA, Crawford J, Aagaard C, et al. (2002). Hypoxic response of Mycobacterium tuberculosis studied by metabolic labeling and proteome analysis of cellular and extracellular proteins. J. Bacteriol. 184: 3485-3491.

Weldingh K, Rosenkrands I, Okkels LM, Doherty TM, et al. (2005). Assessing the serodiagnostic potential of 35 Mycobacterium tuberculosis proteins and identification of four novel serological antigens. J. Clin. Microbiol. 43: 57-65. 